Stability considerations and implementation of cantilevers allowing dynamic force microscopy with optimal resolution: the qPlus sensor

This content has been downloaded from IOPscience. Please scroll down to see the full text. 2004 Nanotechnology 15 S79

(http://iopscience.iop.org/0957-4484/15/2/017)

View the table of contents for this issue, or go to the journal homepage for more

Download details:

IP Address: 132.199.101.133

This content was downloaded on 12/05/2016 at 07:26

Please note that terms and conditions apply. 


\title{
Stability considerations and implementation of cantilevers allowing dynamic force microscopy with optimal resolution: the qPlus sensor
}

\author{
F J Giessibl, S Hembacher, M Herz, Ch Schiller and J Mannhart \\ Universität Augsburg, Experimentalphysik 6, EKM, Institut für Physik, \\ 86135 Augsburg, Germany ${ }^{1}$
}

Received 7 November 2003

Published 22 January 2004

Online at stacks.iop.org/Nano/15/S79 (DOI: 10.1088/0957-4484/15/2/017)

\begin{abstract}
In frequency modulation atomic force microscopy, the stiffness, quality factor and oscillation amplitude of the cantilever are important parameters. While the first atomic resolution results were obtained with amplitudes of a few hundred ångstrom, it has subsequently been shown that smaller amplitudes should result in a better signal-to-noise ratio and an increased sensitivity to the short-range components of the tip-sample interaction. Stable oscillation at small amplitudes is possible if the product of stiffness and amplitude and the energy stored in the oscillating cantilever are large enough. For small amplitudes, stability can be achieved by using stiff cantilevers. Here, we discuss the physical requirements for small amplitude operation and present design criteria and technical details of the qPlus sensor, a self-sensing cantilever with large stiffness that allows small amplitude operation.
\end{abstract}

(Some figures in this article are in colour only in the electronic version)

\section{Introduction}

Today, atomic force microscopy (AFM) with atomic resolution is mostly done using the frequency modulation method, going back to Albrecht et al [1]. Frequency modulation atomic force microscopy (FM-AFM) [1] is now a standard technique that allows the imaging of semiconductors, metals and insulators with true atomic resolution $[2,3]$. In this mode, the cantilever is driven into oscillation by supplying positive feedback. A drive circuit uses the deflection signal of the cantilever and feeds the phase-shifted and amplitude-controlled deflection signal back to an actuator that holds the cantilever. In the first atomic resolution experiment, a cantilever with a spring constant of $17 \mathrm{~N} \mathrm{~m}^{-1}$, an eigenfrequency of $114 \mathrm{kHz}$ and a quality factor of 28000 was operated in the frequency-modulation mode with a constant amplitude of $A=340 \AA$ [5]. The tipsample interaction caused the operating frequency to drop by $70 \mathrm{~Hz}$. The most remarkable factor in this set of empirical parameters is the large oscillation amplitude. The chemical bonding forces responsible for the image contrast have merely a range of about $1 \AA$, and the amplitude is several hundred times greater. Intuitively, it seems that smaller amplitudes should yield better results, and calculations proposed in the first NCAFM workshop in 1998 [6] and subsequent studies [4, 7, 8] predict two advantages of small amplitude operation-reduced noise and increased short-range-force sensibility. For a tipsample force $F_{\text {ts }}$ with range $\lambda$, the signal (frequency shift $\Delta f$ ) is approximately proportional to $1 /\left(1+(\pi / 2)^{1 / 2}(A / \lambda)^{3 / 2}\right)[6]$. Precise analytic equations are available for inverse power force laws [7, 9], power- and exponential laws [7]. For $A \rightarrow 0$ and $A \rightarrow \infty$, the approximative expression given above merges into the accurate analytic formulae. The noise in the frequency measurement has two components: (a) thermal noise and (b) detector noise [1]. Thermal noise varies as $1 / A$ with amplitude [1], as well as detector noise [4, 8, 10]. Thus, a maximum in the signal-to-noise ratio is expected for

$$
A_{\text {opt }} \approx \lambda
$$


A

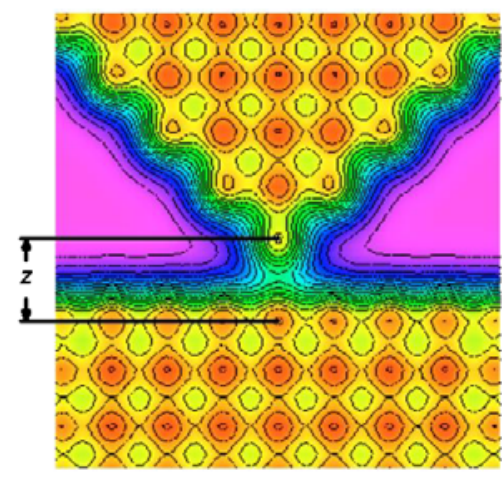

C

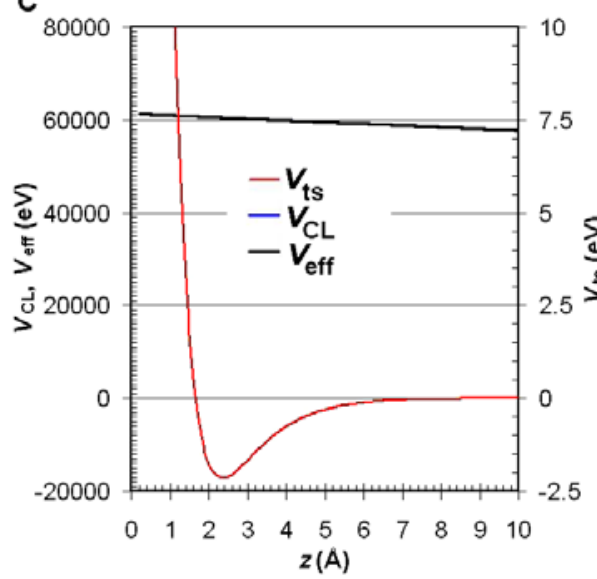

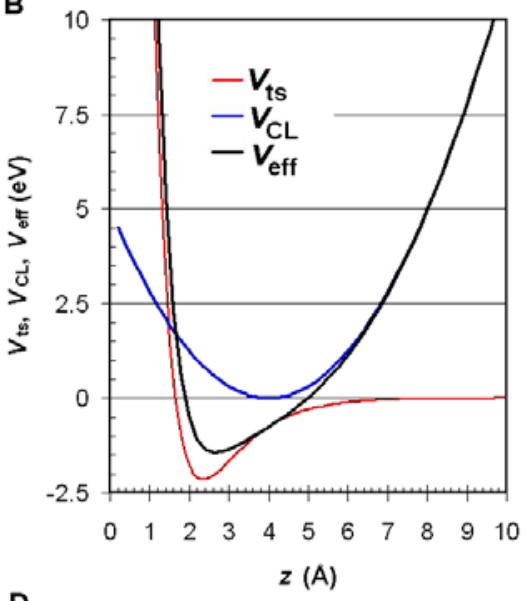

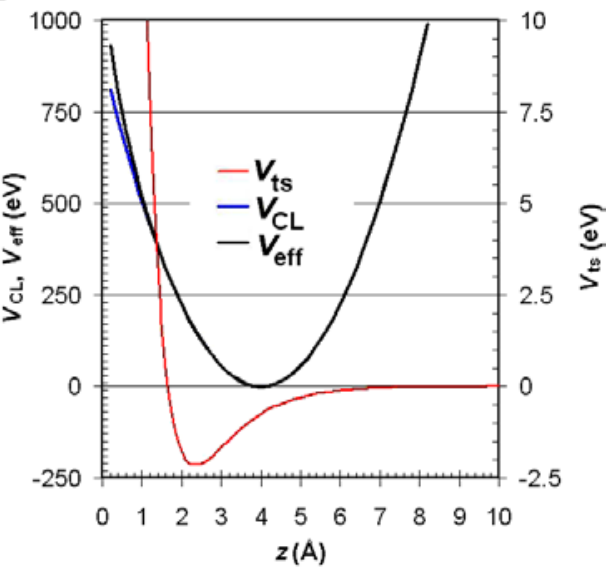

Figure 1. (A) Schematic representation of a sharp tip close to a flat sample. (B) Tip-sample potential, harmonic cantilever potential and effective cantilever potential for a 'soft' cantilever $\left(k=17 \mathrm{~N} \mathrm{~m}^{-1}\right)$. Note that for soft cantilevers, the potential is highly anharmonic. (C) Tip-sample potential, harmonic cantilever potential and effective cantilever potential with $k=17 \mathrm{~N} \mathrm{~m}^{-1}$ is deflected by a large oscillation amplitude (here $A=340 \AA$ ). (D) Tip-sample potential, harmonic cantilever potential and effective cantilever potential for a cantilever with $k=1800 \mathrm{~N} \mathrm{~m}^{-1}$.

(see figure 34 in [4]). The forces responsible for atomic resolution imaging have a range of $\lambda \approx 1 \AA$ [11]. Experiments using relatively soft cantilevers with $k \approx 20 \mathrm{~N} \mathrm{~m}^{-1}$ show that minimal image noise is still obtained for amplitudes of the order of $100 \AA$. We propose that this contradiction is rooted in amplitude fluctuations caused by non-conservative parts of the tip-sample interaction and other instabilities described in the next section. The theoretical calculation of the noise minimum implicitly assumes that the amplitude oscillates at a perfectly constant amplitude. However, dissipative interactions of a magnitude $\delta E_{\text {ts }}$ will cause amplitude fluctuations given by $\delta A=\delta E_{\mathrm{ts}} / k A$ and these amplitude fluctuations directly couple into vertical noise. Also, stable cantilever oscillations that are close to perfect sinusoidal motion are only possible if the anharmonic contributions of the tip-sample interaction are small compared to the harmonic cantilever potential. These stability problems and their solution, a cantilever with sufficient stiffness, are discussed in the following.

\section{Requirements for cantilever stability}

Figure 1(A) shows the tip of a cantilever in close vicinity to a flat sample. The potential between tip and sample can be divided into a short-range component that is dominated by the interaction of the front atom to the sample atom next to it and a long-range contribution that originates from van-der-Waals and possibly electrostatic and magnetic forces [12]. When the tip is mounted on a cantilever, it is also subject to a harmonic cantilever potential $V_{\mathrm{CL}}$. The cantilever potential is a quadratic function of the cantilever's deflection $q^{\prime}$ :

$$
V_{\mathrm{CL}}\left(q^{\prime}\right)=\frac{k}{2} q^{\prime 2}
$$

where $k$ is the spring constant of the cantilever. The tip-sample interaction potential is given by $V_{\mathrm{ts}}(z)$, where $z$ is the distance between the centre of the front atom from the centre of the surface atom next to it. For a silicon tip and a silicon sample, a Morse potential

$$
V_{\mathrm{ts}}(z)=E_{\text {bond }}\left(-2 \mathrm{e}^{-\kappa(z-\sigma)}+\mathrm{e}^{-2 \kappa(z-\sigma)}\right)
$$

is a fair approximation for the short-range part of the tipsample interaction [11]. Here, $E_{\text {bond }}$ is the bonding energy, $\kappa$ is the inverse interaction range and $\sigma$ is the equilibrium distance, for $\mathrm{Si}$ tip and samples, $E_{\text {bond }} \approx 2.15 \mathrm{eV}, \kappa \approx 1 \AA^{-1}$ and $\sigma \approx 2.35 \AA$ [11]. The long-range forces are often even larger in magnitude than the short-range forces and aggravate the stability challenges outlined in the following. 
If the cantilever is placed close to the sample such that the tip-sample distance is $z_{0}$ when the cantilever deflection is zero, the effective potential is given by

$$
V_{\mathrm{eff}}(z)=\frac{k}{2}\left(z_{0}-z\right)^{2}+V_{\mathrm{ts}}(z)
$$

Figure $1(\mathrm{~B})$ shows $V_{\mathrm{ts}}(z)$ defined by equation (3), $V_{\mathrm{CL}}(z)$ for $k=17 \mathrm{~N} \mathrm{~m}^{-1}$ and $V_{\text {eff }}(z)$ for $z_{0}=4 \AA$ defined by equation (4). The effective cantilever potential is strongly anharmonic for this case, and the cantilever's motion would have large unwanted higher-harmonic contributions as calculated by Dürig [13], and it is doubtful if the self-excitation scheme sustaining the cantilever oscillation would still be functional. However, if the same cantilever is placed at $z_{0}=344 \AA$ and oscillates at an amplitude of $A=340 \AA$, the effective potential is almost perfectly harmonic for the $z$-range covered by the oscillating cantilever as shown in figure 1(C). Figure 1(D) shows the potential for a cantilever with a stiffness of $1800 \mathrm{~N} \mathrm{~m}^{-1}$. Even though $z_{0}$ is only $4 \AA$ here as in figure 1(B), $V_{\text {eff }}$ is close to a perfectly harmonic potential.

In FM-AFM, the cantilever's resonance frequency changes with the tip-sample interaction. The free oscillation frequency of the cantilever is given by

$$
f_{0}=\frac{1}{2 \pi} \sqrt{\frac{k}{m^{*}}}
$$

where $m^{*}$ is the effective mass of the cantilever. When the cantilever gets close to the sample, the tip-sample potential $V_{\mathrm{ts}}$ with $k_{\mathrm{ts}}=\partial^{2} V_{\mathrm{ts}} / \partial z^{2}$ has to be taken into account and the resonance frequency changes to

$$
f=\frac{1}{2 \pi} \sqrt{\frac{k+k_{\mathrm{ts}}}{m^{*}}} .
$$

If $k_{\text {ts }}$ is small compared to $k$, the square root can be expanded into a Taylor series and the frequency shift $\Delta f=f-f_{0}$ is given by

$$
\Delta f=f_{0} \frac{k_{\mathrm{ts}}}{2 k} .
$$

If $k_{\mathrm{ts}}$ is not constant for the distance range $z_{0}-A<z<z_{0}+A$ that is covered by the oscillating cantilever, it has to be replaced by an average tip-sample force gradient $\left\langle k_{\mathrm{ts}}\right\rangle$ [14] given by [15]:

$$
\left\langle k_{\mathrm{ts}}\right\rangle=\int_{-A}^{A} k_{\mathrm{ts}}\left(x, y, z+z^{\prime}\right) \frac{\sqrt{A^{2}-z^{\prime 2}}}{\pi A^{2} / 2} \mathrm{~d} z^{\prime} .
$$

These formulae have been obtained with a perturbation approach, which is only valid if the perturbation $\left(V_{\mathrm{ts}}\right)$ is small compared to the harmonic cantilever potential $V_{\mathrm{CL}}$. For strong perturbations, the Taylor series expansion may require the inclusion of higher order terms. In typical FMAFM experiments, the perturbation is small compared to the cantilever potential and the approximations above are fully justified.

Why is it that atomic resolution was initially only obtained using rather large amplitudes? The reason is that the cantilever needs to maintain a stable oscillation while it is scanning the sample. Stability exists if $k$ and $A$ fulfill certain conditions related to $V_{\mathrm{ts}}$ and its derivatives. Two types of instabilities can occur. Tabor and Winterton [16] already realized in 1969 in static force measurements (no scanning), that when measuring the force between two bodies by attaching one of them to a spring with stiffness $k$ and measuring its deflection, an instability can occur. This 'jump-to-contact' instability is prevented if

$$
k>\max \left(-k_{\mathrm{ts}}\right)
$$

where $k_{\mathrm{ts}}=\partial^{2} V_{\mathrm{ts}} / \partial^{2} z$. For a cantilever that oscillates at amplitude $A$, this criterion does not have to be fulfilled as long as

$$
k A>\max \left(-F_{\mathrm{ts}}\right)
$$

for the distance range covered by the oscillating cantilever [17]. Thus, for oscillating cantilevers, jump-to-contact can be avoided even for very soft cantilevers as long as the product of $k$ and $A$ is larger than the maximum of the inverted attractive force $\max \left(-F_{\mathrm{ts}}\right)$. While equation (10) is a necessary condition for stable oscillation, it is not sufficient. If the tip-sample interaction is non-conservative (i.e. a hysteresis in the force versus distance curve), the setup that drives the cantilever into oscillation needs to be able to provide the energy lost within every oscillation cycle.

'Jump-to-contact' causes a complete breakdown of the oscillation. An additional dynamic stability criterion relates to a comparison between the energy losses that occur when the tip of the cantilever approaches and retracts from the sample and the internal energy losses in the cantilever. In the following, we analyse the energetics of constant amplitude control in dissipative environments and discuss their links to the dynamic stability criterion.

FM-AFM involves several nested feedback loops for distance regulation, amplitude control and frequency detection. In a recent study, Gauthier et al [18] performed numerical simulations of the interplay of these feedback circuits. Gauthier et al have found a compelling explanation for lateral shifts of the dissipation signals with respect to the sample topography as a consequence of the time constants within the FM-AFM components. As they point out, the complete analysis of the amplitude control problem involves the solution of a set of complicated integrodifferential equations that can only be tackled by numerical methods. Because of the large number of input parameters, numerical methods do not yield an immediate insight into the sensitivity of the solution to input parameters. Here, we focus on the interplay of amplitude feedback and local variations in the hysteretic part of the tip-sample interaction and look for analytic relations connecting experimental parameters and image noise.

Figure 2 shows the excitation scheme for constantamplitude AFM operation. The input signal of the amplitude regulator is the cantilever deflection output from the cantilever's deflection sensor. This signal is split into two channels. One channel goes into a device that measures the actual amplitude, usually a rms-to-dc converter (e.g. AD536 $\mathrm{A}^{2}$ ). The rms-to-dc converter uses an averaging capacitor or an output filter with a time constant $\tau_{\text {rms-to-dc }}$ in order to provide a smooth output with small ripple $A_{\text {rms }}$ and for enabling precise amplitude measurements. The noise in the amplitude measurement is given by $n_{q^{\prime}} / \sqrt{\tau_{\text {rms-to-dc }}}$,

2 Analog Devices, Norwood, MA, USA, www.analog.com (2003). 


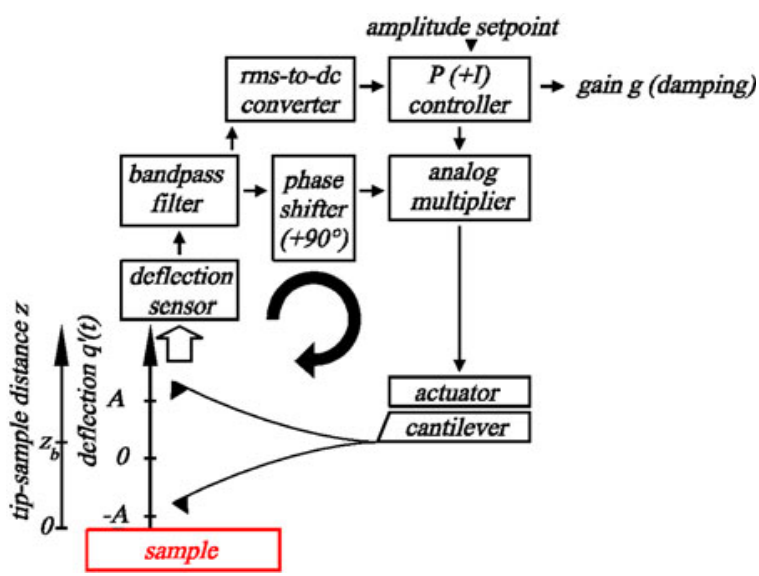

Figure 2. Block diagram of the amplitude regulator in FM-AFM.

where $n_{q^{\prime}}$ is the cantilever deflection noise density. For $n_{q^{\prime}}=100 \mathrm{fm} \mathrm{Hz}^{-1 / 2}, \tau_{\text {rms-to-dc }}$ needs to be at least $10 \mathrm{~ms}$ if the amplitude is to be measured with an accuracy of $0.01 \AA$. Practical values for $\tau_{\text {rms-to-dc }}$ range from $10 / f_{0}$ to $1000 / f_{0}$. In the amplitude regulator, $A_{\text {rms }}$ is divided by the amplitude setpoint $A_{\text {set }}$, and the amplitude error signal $\epsilon_{A}=1-A_{\text {rms }} / A_{\text {set }}$ is input to a $\mathrm{P}$ (proportional) or a PI (proportional + integral) controller to compute the gain signal $g$ that is multiplied by the phase-shifted cantilever deflection signal and fed back to a piezoelectric actuator that excites the cantilever with an amplitude $A_{\text {drive }}$. Further details of the amplitude controller are given in [18] and [8] — here it is only important to note that measuring the actual amplitude involves a settling time $\tau_{\text {rms-to-dc. }}$ A further time delay is caused by the response time of the cantilever. When the driving amplitude of the actuator changes by $\Delta A_{\text {drive }}$, it takes an additional time $\tau_{\mathrm{CL}}=Q /\left(\pi f_{0}\right)$ before the cantilever amplitude settles [1].

Figure 3 shows the flow of energy into and out of the oscillating cantilever. When the cantilever is far from the sample, amplitude control is simple and the dissipation and drive energies are easy to compute. The internal dissipation in the cantilever can be described well by a velocity-dependent friction force given by $-2 \pi f \mathrm{~m}^{*} / Q \mathrm{~d} q^{\prime} / \mathrm{d} t$. For a cantilever that oscillates at frequency $f=1 / T$ according to $q^{\prime}(t)=$ $A \cos (2 \pi t / T)$, the internal energy loss is given by

$$
E_{\text {loss CL }}(t)=\frac{2 \pi E_{0}}{Q}\left(-t / T+\frac{1}{4 \pi} \sin (4 \pi t / T)\right)
$$

if the amplitude is kept constant by driving the cantilever externally, causing an energy loss of $\Delta E_{\mathrm{CL}}=2 \pi E_{0} / Q$ per oscillation cycle, where $E_{0}=k A^{2} / 2$ is the average energy stored in the cantilever and $Q$ is the quality factor. This energy loss is compensated by the automatic gain control unit in the amplitude regulator, which supplies a drive energy

$$
E_{\text {drive }}(t)=\frac{2 \pi E_{0}}{Q}\left(t / T+\frac{1}{4 \pi} \sin (4 \pi t / T)\right) .
$$

The amplitude controller compensates for the internal loss by feeding back energy into the cantilever $\left(E_{\text {drive }}\right.$ in figure 3$)$. If the tip-sample interaction is non-conservative and an additional energy loss of $\Delta E_{\mathrm{ts}}$ per cycle occurs, this energy loss has to be

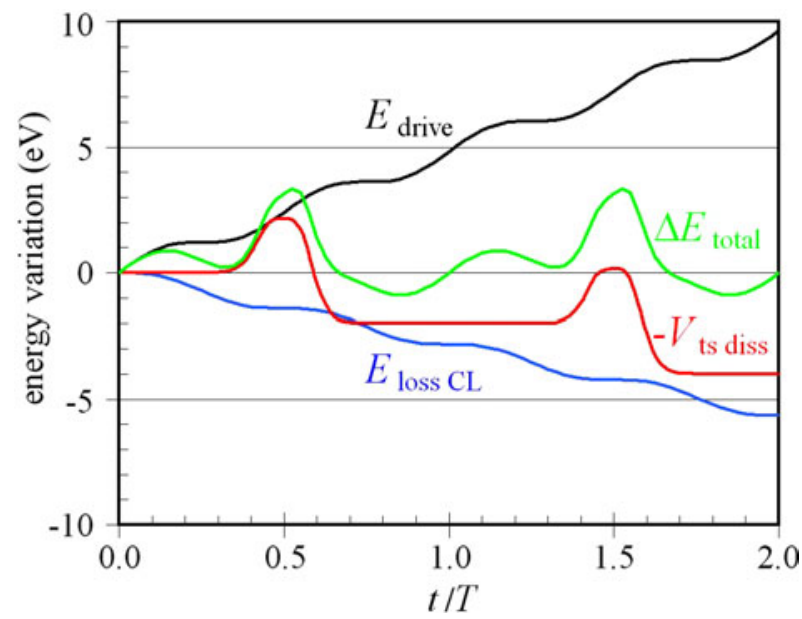

Figure 3. Energy variation versus time for a cantilever that oscillates according to $q^{\prime}(t)=A \cos (2 \pi t / T)$. The total energy of the cantilever is approximately given by $E_{0}=k A^{2} / 2$ with typical values of $1-10 \mathrm{keV}$. The internal friction in the cantilever causes an energy loss proportional to the velocity, indicated by $E_{\text {loss CL. The }}$ tip-sample interaction is usually also dissipative, schematically shown by $-V_{\text {ts diss. }}$. The energy losses $E_{\text {loss CL }}$ and $V_{\text {ts diss }}$ are compensated by feeding back energy $E_{\text {drive }}$ through an actuator. Even if $V_{\mathrm{ts}}$ diss was zero, the total cantilever energy varies with by $\Delta E_{\text {total }}$ with a magnitude given by $E / Q$ around its average value $E_{0}$.

compensated for by a greater energy feedback of the amplitude control circuit.

The first measurements of dissipative interactions in dynamic force microscopy go back to Denk and Pohl [19]. They have measured velocity dependent non-conservative forces between a cantilever with $f_{0}=1 \mathrm{MHz}$ with a resonance width of $300 \mathrm{~Hz}$ and an amplitude of slightly less than $1 \mathrm{~nm}$, thus $\Delta E_{\mathrm{CL}}$ was approximately $0.7 \mathrm{eV}$. They estimated and measured the additional energy loss due to displacement currents that were caused by a tip that was biased by $U_{\mathrm{ts}}=10 \mathrm{~V}$ with a similar magnitude. These damping effects caused by displacement currents are proportional to velocity and the square of $U_{\text {ts }}$. Because $f_{0}$ and $U_{\text {ts }}$ are about one order of magnitude lower in typical FM-AFM experiments, we estimate that the velocity dependent dissipation energy loss is of the order of $1 \mathrm{meV} /$ cycle. A potentially much larger dissipation channel was described by Prandtl in 1928 [20] and one year later [21] by Tomlinson [22] ( 'plucking action of one atom onto an other'). The Prandtl-Tomlinson process is independent of velocity and causes a constant energy loss per cycle $\Delta E_{\mathrm{ts}}$. This dissipation process involves an atomic jump-to-contact. Attaching a strong magnet to a soft coil spring and lowering this arrangement onto a steel plate serves as a model for this dissipation channel. When the magnet approaches the steel plate, it will suddenly snap onto the plate at a fairly close distance. When lifting the spring from the metal plate, the coil spring will stretch until the load force overcomes the attractive force of the magnet, the magnet will snap back from the plate and oscillate until the stored energy dissipates in a damped oscillation. For Si tips and Si samples, Sasaki and Tsukada [23] and Abdurixit et al [24] have performed theoretical studies regarding dissipative interactions. Sasaki and Tsukada have estimated an energy loss comparable to 
the bonding energy of $\approx 2 \mathrm{eV} /$ cycle caused by the PrandtlTomlinson process. Abdurixit et al found an energy loss of approximately $36.5 \mathrm{kcal} \mathrm{mol}^{-1} \approx 1.6 \mathrm{eV} /$ cycle (figure 4 in [24]). An experimental study involving lateral oscillation of a cantilever reports an energy loss of $4 \mathrm{eV}$ per oscillation cycle when the cantilever oscillates between two adatoms [25], thus the energy loss when making and breaking a single bond to $\mathrm{Si}$ is also $2 \mathrm{eV} /$ cycle.

The contributions to energy fluctuations caused by the tipsample interaction are schematically shown in the $-V_{\text {ts diss }}$ graph in figure 3 . When the oscillating cantilever enters the attractive tip-sample potential, it gains a potential energy given by $V_{\mathrm{ts}}\left(z_{0}+A \cos (2 \pi t / T)\right)$ defined by equation (3) for $0<t<T / 2$. In figure $3, z_{0}=A+\sigma$ and the cantilever has gained the energy $E_{\text {bond }}$ of equation (3) at its closest sample encounter at $t=T / 2$. If a Prandtl-Tomlinson loss process occurs, the attractive potential requires an energy of $\Delta E_{\mathrm{ts}}+E_{\mathrm{bond}}$ as shown by the section of the $-V_{\mathrm{ts}}$ diss graph of figure 3 for $T / 2<t<T$. This extra energy loss occurs at every consecutive oscillation cycle and has to be compensated for by the amplitude controller. If the energy losses due to nonconservative tip-sample potentials are constant or changing very slowly, amplitude control is not a problem. However, if $\Delta E_{\mathrm{ts}}$ varies strongly from one atomic position to the other, the oscillation amplitude is affected. What happens to the cantilever amplitude when the tip scans from a position with almost zero tip-sample dissipation to an atomic position that causes a dissipation of a few electronvolt per cycle? Because the amplitude controller responds slowly as outlined above, the energy feeding the dissipation channels has to come from the energy stored in the cantilever, leading to a change of its amplitude from $A$ to $A^{\prime}$ :

$$
\frac{1}{2} k A^{\prime 2}=\frac{1}{2} k A^{2}-\Delta E_{\mathrm{ts}}
$$

Thus, for a time given by $\tau_{\text {rms-to-dc }}$, the amplitude will drop by $\Delta A=A^{\prime}-A$ per cycle with

$$
\Delta A=\sqrt{A^{2}-2 \Delta E_{\mathrm{ts}} / k}-A \approx \frac{\Delta E_{\mathrm{ts}}}{k A}
$$

with little change in the measured cantilever amplitude $A_{\mathrm{rms}}$ because of the time constant of the rms-to-dc converter. In addition, we have to take into account that changes in the driving amplitude $A_{\text {drive }}$ are reflected in changes of $A$ after a time delay given by $\tau_{\mathrm{CL}}=Q /\left(\pi f_{0}\right)$ and thus require approximately $Q / \pi$ oscillation cycles before $A$ reaches its original setpoint again. If we demand that the cantilever is still oscillating after $Q / \pi$ cycles with extra damping present, we find

$$
\Delta A \frac{Q}{\pi}<A \Rightarrow \Delta E_{\mathrm{ts}}<\frac{\pi k A^{2}}{Q} .
$$

We have neglected the slow buildup of the driving amplitude by the amplitude controller, therefore equation (15) is only an approximative criterion. Reductions in amplitude need to be minimized because they will also lead to a change in the frequency shift, leading to a coupling of the distance regulator and the amplitude controller. It is interesting to note that the conclusion in equation (15) is exactly the same criterion that was proposed as a conjecture in $[4,8]$. In the earlier derivation, the conclusion in equation (15) was based on the requirement that the gain factor in the amplitude controller should not vary by more than $100 \%$ from its value when the sample is far from the oscillating tip. Finally, it is important to add a short discussion about the optimal value of the cantilever's quality factor $Q$. One implication of equation (15) is that the $Q$-factor should not be too large. This appears to be in contradiction with the findings of Albrecht et al [1] that the noise in the frequency measurement in AFM is proportional to $1 / \sqrt{Q}$. However, it has to be considered that the damping of the cantilever and thus its $Q$-value change when dissipative sample interaction is present. Since $Q$ is given by $2 \pi$ times the energy stored in the cantilever divided by the energy loss per cycle, the effective $Q$ value is

$$
Q_{\text {eff }}=\frac{1}{\frac{1}{Q}+\frac{\Delta E_{\mathrm{ts}}}{2 \pi E_{0}}} .
$$

Thus $Q_{\text {eff }}$ does not increase much if $Q$ is chosen to be larger than $\frac{2 \pi E_{0}}{\Delta E_{\mathrm{ts}}}$ and values of $Q \approx 5000$ should be sufficient for most practical applications.

\section{The qPlus sensor}

By the time we realized the potential benefits of small amplitude operation and the requirement of very stiff cantilevers with $k \approx 1 \mathrm{kN} \mathrm{m}^{-1}$, standard silicon cantilevers with such a high stiffness were not available. This was a good opportunity to take a fresh look at the problem of minimizing noise in frequency modulation AFM. Equation (7) connects the measurement of small forces to a measurement of frequencies. The measurement of time and frequency has been pursued by 'physicists' for at least a few millennia and research into chronology with its implications for navigation and land surveying is probably the first example for large-scale government funding of fundamental research [26]. With the invention of atomic clocks, of all physical measurements time and frequency can be measured with the greatest precision. While it is doubtful that utilizing atomic clocks in force microscopy is practical, it is worthwhile to look at the second best solution for frequency standards: quartz oscillators. Unlike silicon with its cubic crystal symmetry, quartz is highly anisotropic with a hexagonal crystal symmetry. The variation of the oscillation frequency of mechanical oscillators made of quartz depends on the crystal orientation and a number of crystal orientations with low temperature dependence have been found [27]. For tuning fork geometries, the ' $X+5^{\circ}$ cut' [28] has a very low temperature variation given by $\Delta f / f \approx \zeta\left(\vartheta-\vartheta_{0}\right)^{2}$ with $\zeta=-3.5 \times 10^{-8} /{ }^{\circ} \mathrm{C} \pm 10 \%$ and $\vartheta_{0}=25^{\circ} \mathrm{C} \pm 5{ }^{\circ} \mathrm{C}{ }^{3}$ In frequency modulation AFM, thermal variations of cantilever frequency are a source of noise. Figure 4 shows the frequency shift coefficient of quartz and silicon cantilevers. The quartz data are fitted to experimental measurements published in [29], the silicon data is calculated from the materials properties of silicon in the [110] crystal direction [30]. For a temperature range of room temperature $\pm 10^{\circ} \mathrm{C}$, the frequency of a standard silicon cantilever (cantilever extends along the [110] crystal direction) with $f_{0}=100 \mathrm{kHz}$ would change by $\pm 35 \mathrm{~Hz}$, while the frequency of a quartz cantilever $\left(X+5^{\circ}\right.$ cut) would change

\footnotetext{
3 Micro Crystal, Mühlestrasse 14, CH-2540 Grenchen, Switzerland,
} www.microcrystal.ch (2002). 


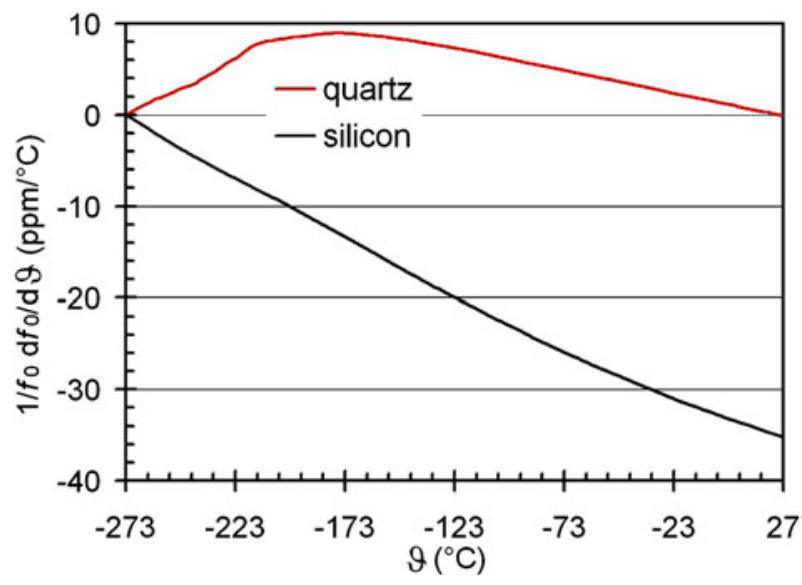

Figure 4. Frequency shift coefficient as a function of temperature for quartz and silicon cantilevers (see text).

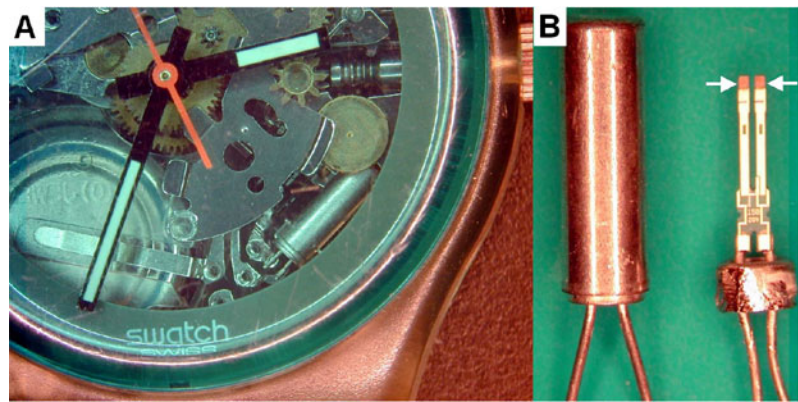

Figure 5. (A) Transparent quartz watch, (B) time-keeping element of the watch; a quartz tuning fork in an evacuated metal case (left) and opened case (right)

only by $-0.35 \mathrm{~Hz}$. For operation at liquid helium temperatures, the magnitude of the frequency shift with temperature is comparable for $\mathrm{Si}[110]$ and quartz. However, it has to be noted that the deflection of traditional Si cantilevers has to be measured by optical means, typically shining light onto the cantilever with a power of the order of $1 \mathrm{~mW}$, so that the actual cantilever temperature might be considerably higher than the temperature of the other parts of the microscope. With piezoelectric detection, the energy losses due to deflection detection are a few orders of magnitude lower, therefore lower cantilever temperatures are expected for quartz sensors with piezoelectric detection.

Figure 5(A) shows a transparent SWATCH ${ }^{\mathrm{TM}}$ wristwatch where the metal can that holds the tuning fork is clearly visible. Figure 5(B) shows a magnified view of an enclosed (left) and an open tuning fork (right). The whole tuning forks have a length of $4 \mathrm{~mm}$, the individual prongs have a length of $2.4 \mathrm{~mm}$ (vertical in figure 5(B)), a thickness of $214 \mu \mathrm{m}$ (horizontal) and a width of $130 \mu \mathrm{m}$ (perpendicular to the paper plane). In the mounted version shown in figure 5(B), the gold metallization is removed in a rectangular region at the electrically inactive end sections of the prongs and a fine line below by laser trimming (indicated by the arrows in figure 5(B), compare with figure 6(A) for reference). This laser trimming reduces the effective mass of the prongs and tunes them to an eigenfrequency of exactly $2^{15} \mathrm{~Hz}$. We have measured the thickness of the gold layer to be $220 \mathrm{~nm}$ and estimate a laser spot size of roughly $(10 \mu \mathrm{m})^{2}$, thus the prongs are trimmed with

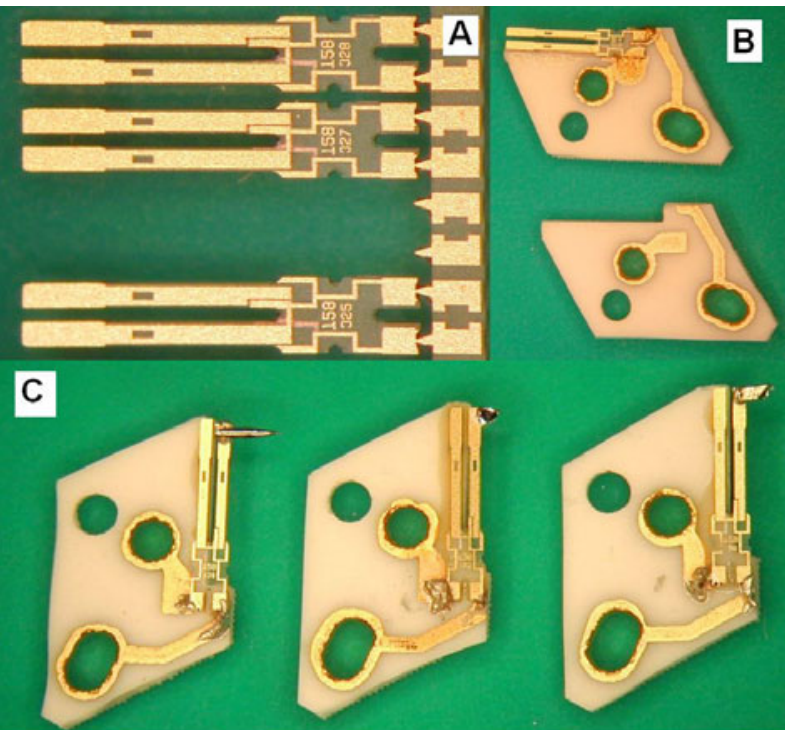

D

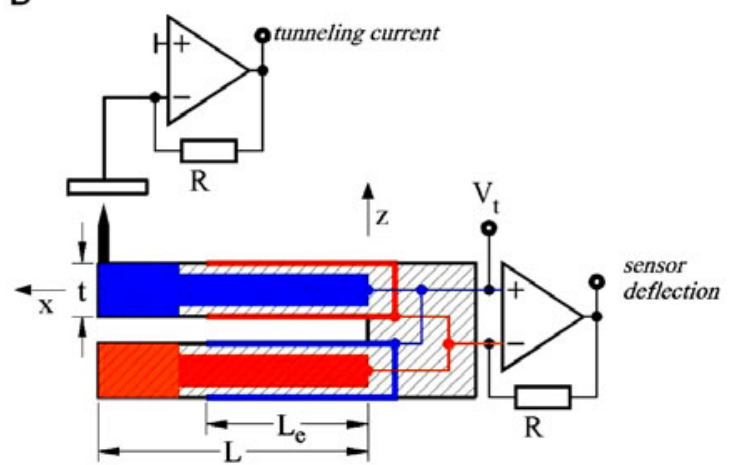

Figure 6. (A) Section of a wafer of tuning forks type E158 from (see footnote 3). (B) Alumina substrate (bottom) and tuning fork glued to the substrate (top). (C) Three assembled qPlus sensors with tips made from tungsten (left), silicon (centre) and iridium (right). (D) Wiring and electronic scheme for simultaneous STM and AFM operation with qPlus sensor.

a precision of $\delta m / m^{*}=10^{-7}$ corresponding to a frequency precision of $3 \mathrm{mHz}$. The prongs form two coupled oscillator and the mode with the highest $Q$-factor is the antiparalle motion where both prongs move in opposite directions. The tuning forks we use have a quality factor of $Q \approx 55000$ when they are enclosed in the evacuated metal can (see footnote 3 ). If the eigenfrequency of the two prongs does not match, a ringing mode with low $Q$ results. Quartz watch crystals in tuning fork geometry have been used before in acoustic [31] and optical near field microscopy [32]. In these first applications of tuning forks as force sensors, a tip was attached to one of the prongs, resulting in an arrangement where the prong that carries the probe has a significantly lower eigenfrequency. To solve the problem of the resulting ringing mode, Dransfeld et al [33] have proposed attaching a counterweight on the prong without a tip. However, tip-sample interactions also break the symmetry of the two prongs. In the 'qPlus' configuration, one prong is fixed to a substrate with high mass, rendering the tuning fork into a cantilever geometry. The advantage is that $Q$ remains constant even when the eigenfrequency of the free prong changes due to (conservative) tip-sample interactions. 
The quality of the attachment of the tuning forks to the alumina substrate is critical for obtaining high $Q$ values. We attach the forks to the substrate with Torr-Seal, a vacuum compatible epoxy $\operatorname{resin}^{4}$ and obtain a $Q$ value of about 4000 in vacuum at room temperature and 20000 in vacuum at liquid helium temperature [34]. King et al [35] use a different type of glue and obtain a $Q$ value of about 5000 in air at room temperature.

Figure 6(A) depicts a section of a wafer of tuning forks of the type we are using (E158 as used in crystals DS26 from Micro Crystal (see footnote 3)). Figure 6(B) displays a custom made alumina substrate (bottom) and a tuning fork glued to it (top), and figure 6(C) three assembled qPlus sensors with tips made from tungsten (left), silicon (centre) and iridium (right). In the unmounted tuning forks shown in figure 6(A), the gold metallization in the end sections of the prongs is a closed layer.

Figure 6(D) shows the electronic circuitry for simultaneous STM/AFM operation. The tuning forks have two electrodes. The electrode at the end of the free prong connects the tip of the sensor to the tip bias voltage. The other electrode is connected to a current-to-voltage converter-the output of this circuit is proportional to the derivative of the sensor deflection with respect to time [36]. The tunnelling current is collected at the sample. Both current-to-voltage converters should have a high bandwidth and low noise (e.g. AD711 or AD744 from (see footnote 2)), using resistors with a low parasitic capacitance and typical values of $100 \mathrm{M} \Omega$.

In order to make full use of the high frequency stability of the sensor, we use a digital, quartz stabilized phase-lockedloop detector ${ }^{5}$.

In summary, the qPlus sensor has four additional benefits over standard silicon cantilevers:

(1) Tuning forks are available with a stiffness close to the desired value of $1 \mathrm{kN} \mathrm{m}^{-1}$.

(2) The forks are large, allowing the mounting of various types of tips on them. Most tip preparation methods known from scanning tunnelling microscopy can be utilized. The large size also allows the sensor to be oriented parallel to the sample surface and oscillate it exactly perpendicular to the sample surface - traditional silicon cantilevers have to be tilted by about $10^{\circ}$. Also, the qPlus sensor can be tilted by $90^{\circ}$ to allow lateral force detection [25].

(3) Quartz, the material of the tuning forks is far superior to silicon with respect to frequency stability.

(4) Because of the piezoelectricity of quartz, the deflection of quartz cantilevers produces charges at electrodes on the surfaces of the oscillators. The deflection measurement is easily done electrically without the need for optical components, simplifying the implementation considerably.

\section{Discussion}

In summary, we have confirmed a previously suggested stability criterion stating that within one oscillation cycle, the intrinsic energy loss in the cantilever should be comparable in

4 Varian Torr Seal Varian, Inc., Hansen Way, Palo Alto, CA 94304-1030, USA (2003).

${ }^{5}$ Nanosurf easyPLL, easyPLL Sensor Controller Nanosurf AG, Grammetstraße 14, CH-8804 Liestal, Switzerland (2003). magnitude to the tip-sample dissipation $\Delta E_{\mathrm{ts}}$. Empirically, this criterion is fulfilled in most atomic resolution experiments (see table 1 in [4]). Exceptions are low-temperature experiments [37, 38] with extremely low scanning speeds, and spectroscopy experiments where the $z$-distance is varied very slowly [39]. If $\Delta E_{\mathrm{ts}}$ varies sufficiently slowly with time, the amplitude regulator has enough time to adjust to strong variations in dissipation.

Meeting this criterion also means decreasing $\Delta E_{\mathrm{ts}}$. It is likely that not only the front atom of the tip, but in particular tip atoms close to the apex that are loosely bonded to the tip, contribute to $\Delta E_{\mathrm{ts}}$. The smallest amplitudes where stable imaging was performed were obtained with tips that were annealed at high temperature. Eguchi and Hasegawa [10] have performed experiments with $A=28 \AA$ with a cantilever with $k=48 \mathrm{~N} \mathrm{~m}^{-1}$ that was annealed at $900{ }^{\circ} \mathrm{C}$, and we have reached amplitudes down to $A=2.5 \AA$ with a qPlus sensor with $k=1800 \mathrm{~N} \mathrm{~m}^{-1}$ where the Si tip was heated close to the melting temperature [40]. We therefore speculate that hightemperature annealing of tips removes weakly bound atoms that are expected to favour the Prandtl-Tomlinson dissipation process.

Because $\Delta E_{\mathrm{ts}}$ increases strongly with decreasing tipsample distance [41-43], imaging at very small tip-sample distances is only possible if the cantilever oscillation remains stable for large $\Delta E_{\mathrm{ts}}$. On the other hand, the resolution of scanning probe microscopes increases with smaller tipsample distances [44-46]. Probing samples at very small tipsample distances thus requires the use of very stiff cantilevers with spring constants of the order of $1 \mathrm{kN} \mathrm{m}^{-1}$. The qPlus sensor is an implementation of such a stiff cantilever and has been described in detail above. Using this stiff sensor, 'subatomic' structures, attributed to two $3 \mathrm{sp}^{3}$-like orbitals originating from a single atom could be made visible [40, 45]. Also, subatomic features in a single atom, attributed to 4 f-like states on Sm [47] could be imaged using this technique. Small amplitude operation also simplifies combined STM/AFM imaging, enabling experiments where both $\alpha$ - and $\beta$-atoms in graphite could be made visible in the force signal while simultaneously imaging the $\beta$-atoms in the current signal [34] using a $5 \mathrm{~K}$ STM/AFM. The small amplitude/stiff cantilever technique has also enabled true atomic resolution in lateral force microscopy [25].

In principle, increased resolution should also be possible with conventional silicon cantilevers and optical detection. We therefore suggest to perform experiments with conventional FM-AFMs using cantilevers with much greater stiffness. In contrast to self-sensing arrangements like the qPlus sensor and piezoresistive cantilevers [48], conventional optical detection does not gain in signal-to-noise ratio of the cantilever deflection measurement with increasing cantilever stiffness. However, the decrease in force sensitivity should be greatly offset by the expected gain in resolution, in particular for low-temperature microscopes where detector noise can be minimized by low scanning speeds and bandwidth reductions.

\section{Acknowledgment}

This work is supported by the Bundesministerium für Bildung und Forschung (project EKM13N6918). 


\section{References}

[1] Albrecht T R, Grutter P, Horne H K and Rugar D 1991 J. Appl. Phys. 69668

[2] Morita S, Wiesendanger R and Meyer E (ed) 2002 Nanoscience and technology Noncontact Atomic Force Microscopy (Berlin: Springer)

[3] Garcia R and Perez R 2002 Surf. Sci. Rep. 47197

[4] Giessibl F J 2003 Rev. Mod. Phys. 75949

[5] Giessibl F J 1995 Science 26768

[6] Giessibl F J, Bielefeldt H, Hembacher S and Mannhart J 1999 Appl. Surf. Sci. 140352

[7] Giessibl F J and Bielefeldt H 2000 Phys. Rev. B 619968

[8] Giessibl F J 2002 Noncontact Atomic Force Microscopy ed S Morita, R Wiesendanger and E Meyer (Berlin: Springer) chapter 2, pp 11-46

[9] Hölscher H, Allers W, Schwarz U D, Schwarz A and Wiesendanger R 1999 Appl. Surf. Sci. 140344

[10] Eguchi T and Hasegawa Y 2002 Phys. Rev. Lett. 89266105

[11] Perez R, Stich I, Payne M C and Terakura K 1998 Phys. Rev. B 5810835

[12] Israelachvili J 1991 Intermolecular and Surface Forces 2nd edn (London: Academic)

[13] Dürig U 2000 New J. Phys. 25.1

[14] Dürig U 1999 Surf. Interface Anal. 27467

[15] Giessibl F J 2001 Appl. Phys. Lett. 78123

[16] Tabor D and Winterton R H S 1969 Proc. R. Soc. A 312435

[17] Giessibl F J 1997 Phys. Rev. B 5616010

[18] Gauthier M, Perez R, Arai T, Tomitori M and Tsukada M 2002 Phys. Rev. Lett. 89146104

[19] Denk W and Pohl D W 1991 Appl. Phys. Lett. 592171

[20] Prandtl L 1928 Z. Angew. Math. Mech. 885

[21] Müser M H 2003 Phys. J. 243

[22] Tomlinson G A 1929 Phil. Mag. 7905

[23] Sasaki N and Tsukada M 2000 Japan. J. Appl. Phys. 39 L1334

[24] Abdurixit A, Baratoff A and Meyer E 1999 Appl. Surf. Sci. 157 355

[25] Giessibl F J, Herz M and Mannhart J 2002 Proc. Natl. Acad. Sci. USA 9912006

[26] Sobel D 2000 The Illustrated Longitude (New York: Walter Books)

[27] Brice J C 1985 Rev. Mod. Phys. 57105
[28] Walls F L 1985 Precision Frequency Control ed E Gerber and A Ballato (Orlando, FL: Academic) pp 276-9

[29] Hembacher S, Giessibl F J and Mannhart J 2002 Appl. Surf. Sci. 188445

[30] Landolt-Börnstein 1982 Numerical Data and Functional Relationships in Science and Technology vol 17a, ed O Madelung, M Schultz and H Weiss (Berlin: Springer)

[31] Guethner P, Fischer U C and Dransfeld K 1989 Appl. Phys. B 4889

[32] Karrai K and Grober R D 1995 Appl. Phys. Lett. 661842

[33] Dransfeld K, Fischer U, Güthner P and Heitmann K 1991 US Patent 5212987

[34] Hembacher S, Giessibl F J, Mannhart J and Quate C F 2003 Proc. Natl Acad. Sci. USA 10012539

[35] King G M, Lamb J S and Nunes J G 2001 Appl. Phys. Lett. 79 1712

[36] Giessibl F J 2000 Appl. Phys. Lett. 761470

[37] Lantz M, Hug H J, van Schendel P, Hoffmann R, Martin S, Baratoff A, Abdurixit A and Güntherodt H-J 2000 Phys. Rev. Lett. 842642

[38] Lantz M, Hug H J, Hoffmann R, van Schendel P, Kappenberger P, Martin S, Baratoff A and Güntherodt H-J 2001 Science 2912580

[39] Pfeiffer O, Nony L, Bennewitz R, Baratoff A and Meyer E 2004 Nanotechnology 15 S101-7

[40] Giessibl F J, Bielefeldt H, Hembacher S and Mannhart J 2001 Ann. Phys. (Berlin) $\mathbf{1 0} 887$

[41] Lüthi R, Meyer E, Bammerlin M, Baratoff A, Howald L, Gerber C and Güntherodt H-J 1997 Surf. Rev. Lett. 41025

[42] Ueyama H, Sugawara Y and Morita S 1998 Appl. Phys. A 66 S295

[43] Bammerlin M, Lüthi R, Meyer E, Baratoff A, Lü J, Guggisberg M, Gerber C, Howald L and Güntherodt H J 1997 Probe Microsc. 13

[44] Stoll E 1984 Surf. Sci. 143 L411

[45] Giessibl F J, Hembacher S, Bielefeldt H and Mannhart J 2000 Science 289422

[46] Huang M, Cuma M and Liu F 2003 Phys. Rev. Lett. 90256101

[47] Herz M, Giessibl F J and Mannhart J 2003 Phys. Rev. B 68 045301

[48] Tortonese M, Barrett R C and Quate C 1993 Appl. Phys. Lett. 62834 